\title{
High prevalence of Lynx rufus gammaherpesvirus 1 in wild Vermont bobcats
}

\author{
Dagan A. Loisel ${ }^{\text {Corresp., }}{ }^{2}$, Ryan M. Troyer ${ }^{2}$, Sue VandeWoude ${ }^{3}$ \\ 1 Department of Biology, St. Michael's College, Colchester, VT, United States \\ 2 Department of Microbiology \& Immunology, University of Western Ontario, London, Ontario, Canada \\ 3 Department of Microbiology, Immunology, and Pathology, Colorado State University, Fort Collins, Colorado, United States \\ Corresponding Author: Dagan A. Loisel \\ Email address: dloisel@smcvt.edu
}

Gammaherpesviruses are host specific DNA viruses that infect a large range of mammalian species. These viruses preferentially target host lymphocyte cell populations and infection may lead to morbidity or mortality in immunocompromised, co-infected, or non-adapted hosts. In this study, we tested for the presence of Lynx rufus gammaherpesvirus 1 (LruGHV1) in a northeastern United States population of wild bobcats (Lynx rufus). We estimated prevalence of infection and viral load in infected individuals using quantitative real-time PCR analysis of spleen DNA from 64 Vermont bobcats. We observed an overall prevalence of $64 \%$ using this methodology. Bobcat age was significantly positively associated with GHV infection status, and we noted a trend for higher viral loads in young animals, but prevalence and viral load were similar in male and female bobcats. A single LruGHV1 variant was identified from the sequencing of the viral glycoprotein $B$ gene of Vermont bobcats. This gene sequence was $100 \%$ similar to that reported in Florida bobcats and slightly variant from other strains identified in the Western US. Our work suggests broad geographic distribution and high prevalence of LruGHV1 in bobcat populations across the United States with infection attributes that suggest horizontal transmission of the agent. Geographic differences in viral genotype may reflect historical migration and expansion events among bobcat populations. 
1 TITLE: High prevalence of Lynx rufus gammaherpesvirus 1 in wild Vermont bobcats

2 AUTHORS: Dagan A Loisel ${ }^{1 *}$, Ryan M. Troyer ${ }^{2}$, Sue VandeWoude ${ }^{3}$

3

$4 \quad{ }^{1}$ Department of Biology, Saint Michael's College, Colchester, VT, USA

$5 \quad{ }^{2}$ Department of Microbiology \& Immunology, Western University, London, Ontario, CA

$6 \quad{ }^{3}$ Department of Microbiology, Immunology, and Pathology, Colorado State University, Fort

7 Collins, CO, USA

$8 \quad$ *Corresponding author: Dagan Loisel, Ph.D.

9 Department of Biology

10 Saint Michael's College

11 One Winooski Park, Box 283

12 Colchester, VT 05439

13 Phone: (802) 654-2259

14 Email: dloisel@smcvt.edu 
16 Abstract

17

18

19

20

21

22

23

24

Gammaherpesviruses are host specific DNA viruses that infect a large range of mammalian species. These viruses preferentially target host lymphocyte cell populations and infection may lead to morbidity or mortality in immunocompromised, co-infected, or non-adapted hosts. In this study, we tested for the presence of Lynx rufus gammaherpesvirus 1 (LruGHV1) in a northeastern United States population of wild bobcats (Lynx rufus). We estimated prevalence of infection and viral load in infected individuals using quantitative real-time PCR analysis of spleen DNA from 64 Vermont bobcats. We observed an overall prevalence of $64 \%$ using this methodology. Bobcat age was significantly positively associated with GHV infection status, and we noted a trend for higher viral loads in young animals, but prevalence and viral load were similar in male and female bobcats. A single LruGHV1 variant was identified from the sequencing of the viral glycoprotein B gene of Vermont bobcats. This gene sequence was $100 \%$ similar to that reported in Florida bobcats and slightly variant from other strains identified in the Western US. Our work suggests broad geographic distribution and high prevalence of LruGHV1 in bobcat populations across the United States with infection attributes that suggest horizontal transmission of the agent. Geographic differences in viral genotype may reflect historical migration and expansion events among bobcat populations. 


\section{Introduction}

Herpesviruses are large, enveloped, double-stranded DNA viruses capable of both lytic (i.e. productive) and latent infection of their hosts (Speck \& Ganem 2010). This ability to express true latency results in lifelong infection punctuated by episodes of reactivation and productive infection in response to environmental stimuli. Herpesviruses are classified into three subfamilies - alpha, beta, and gamma - based on differences in genomic sequence, tissue tropism, host range, initial form of infection, and other biological properties. Gammaherpesviruses (GHVs) are highly host specific and typically cause persistent, inapparent, latent infections in lymphocytes (Speck \& Ganem 2010). GHV-associated disease is primarily observed in immunocompromised hosts as a consequence of latent infection, and to a lesser extent, reactivation of lytic infection (Ackermann 2006; O'Toole \& Li 2014). In humans, latent infection with GHVs, such as EpsteinBarr virus (EBV) and Karposi sarcoma-associated herpesvirus (KSHV), may lead to a variety lymphoproliferative disorders (LPDs) in individuals with impaired immunity due to HIV infection and AIDS, therapeutic immunosuppression, or congenital immunodeficiency (Cesarman 2011; Cesarman 2014).

In animals, GHV infection is generally asymptomatic when found in its natural host, but can lead to pathogenic LPDs in the case of host immune dysfunction, immunodeficiency, and/or coinfection with another virus (Ackermann 2006; Kaye et al. 2016). In domestic cats, the probability of GHV1 detection and associated viral load was higher in cats infected with the pathogenic lentivirus, feline immunodeficiency virus, or in cats that were presented for other illnesses (Beatty et al. 2014; Ertl et al. 2015; Tateno et al. 2017). In addition, GHV transmission to a heterologous or non-adapted host may result in disease. For example, cattle exhibit the fatal lymphoproliferative disease, malignant catarrhal fever, as a consequence of infection with 
58 wildebeest or sheep GHVs that cause inapparent infection in their natural hosts (Russell et al. 59 2009).

60

61

62

Recently, novel GHVs were discovered circulating in North American populations of two wild felids, bobcats (Lynx rufus) and pumas (Puma concolor) (Lozano et al. 2015; Troyer et al. 2014). Two strains, LruGHV1 and LruGHV2, were identified in wild bobcats, and interestingly, the common bobcat strain (LruGHV1) was also detected in wild pumas, in addition to a strain that was puma-specific (PcoGHV1) (Troyer et al. 2014). Furthermore, five polymorphic sites in the LruGHV1 glycoprotein $B(\mathrm{~g} B)$ gene were observed in comparisons of sequences from Florida and California bobcats, suggesting population-specific differences in viral evolution (Troyer et al. 2014). In the same study, a third felid GHV, FcaGHV1, was identified in North American domestic cats (Felis catus) but not wild felids (Troyer et al. 2014). Subsequent studies documented FcaGHV1 DNA in cats sampled in Australia, Europe, Asia, and the US, suggesting a worldwide distribution of this potentially pathogenic virus (Beatty et al. 2014; Ertl et al. 2015; McLuckie et al. 2016; Tateno et al. 2017). Interestingly, the vast majority of domestic cats infected with FcaGHV1 have been male (Beatty et al. 2014; Ertl et al. 2015; McLuckie et al. 2016; Tateno et al. 2017). Serologic analysis determined that approximately half of domestic cats that harbored FcaGHV1 specific antibodies did not have detectable DNA in circulation, suggesting that prevalence estimates based on the detection of FcaGHV1 DNA may be understating the extent of infection (Stutzman-Rodriguez et al. 2016). A causal role for FcaGHV1 in the development of lymphomas or other diseases in domestic cats has been hypothesized, but not yet demonstrated.

79 In this study, we investigated the presence and prevalence of GHV in a wild population of bobcats in the northeast United States. We used quantitative real-time PCR (qPCR) to detect 
81 GHV DNA in spleen samples from Vermont bobcats in order to estimate prevalence of GHV

82 infection in the population and viral load in infected individuals. We then sequenced a segment

83 of the GHV $g B$ gene in infected bobcats to determine GHV strain identity and to characterize

84 potential population-specific genetic variants. Finally, we tested for an association between the

85 bobcat demographic traits, age and sex, and viral infection status to better understand the

86 transmission and ecology of the virus. 


\section{Materials \& Methods}

88 Sample collection

89

90

91

92

93

94

95

96

97

The protocol to collect animal samples was approved prior to collection by the Saint Michael's College Institutional Animal Care and Use Committee under protocol \#022-2016. Spleen samples were obtained from bobcat carcasses collected by the State of Vermont Fish and Wildlife Department during the 2015 (1/29/15-12/15/15) and $2016(1 / 11 / 16-2 / 5 / 17)$ seasons. Carcasses showing severe autolysis were not sampled. Immediately after collection, spleen samples were assigned unique alphanumeric ID numbers, transferred to cryogenic storage tubes containing RNAlater stabilization solution (Ambion by Life Technologies, Carlsbad, CA), and stored at -20C temporarily, and at -80C permanently in the Biology Department at Saint Michael's College. Spleen tissue was chosen for study in order to maximize the probability of detecting GHV based on previous studies documenting the highest prevalence in spleen compared to other tissues and blood (Beatty et al. 2014; Lozano et al. 2015) and the observation that GHVs are more readily detected during latency in organs, such as the spleen, containing a significant B lymphocyte cell population (Coleman et al. 2010).

Information about bobcat age, sex, and approximate location of collection (i.e. city or town) was provided by the Vermont Fish and Wildlife Department. Age of individual bobcats was estimated from a species-specific standardized aging model based on the number of cementum annuli observed in Giemsa-stained histological sections of a lower canine tooth, as performed by Matson's Laboratory (Manhattan, MT) using methodology described previously (Boertje et al. 2015). Age categories were defined as follows: young bobcats correspond to age estimates up to 2 years of age and adult bobcats correspond to age estimates greater than or equal to 2 years of age. Spleen samples from the 2015 and 2016 seasons that had associated age, sex, 
110 and collection location data were included in this study. Of the 64 samples meeting that criteria,

$11148.4 \%$ were derived from bobcats harvested by licensed trappers, 37.50\% were derived from

112 bobcats harvested by licensed hunters, $12.5 \%$ were collected from dead bobcats after motor

113 vehicle collisions, and 1.6\% had unknown provenance, according to the Vermont Fish and

114 Wildlife Department.

115 DNA extraction and quantification

116 Total DNA was extracted from spleen tissue samples ( 400 milligrams each) using the

117 manufacturer's recommended protocol for the Gentra Puregene Tissue Kit (Qiagen, Inc.,

118 Valencia, CA). After extraction, the concentration (ng/ $\mu$ l) and purity (260/280 ratio) of DNA in

119 each sample was determined using a NanoDrop 2000 spectrophotometer (Thermo Fisher

120 Scientific, Wilmington, DE). DNA samples were diluted to $100 \mathrm{ng} / \mu 1$ working stocks for

121 subsequent genetic analyses.

122 Quantitative real-time PCR (qPCR)

123 The glycoprotein B gene of LruGHV1 was targeted for amplification using primers and 5'

124 6-carboxyfluorescein (FAM) labeled and 3' 6-carboxytetramethylrhodamine (TAMRA) labeled

125 probe sets as described previously (Troyer et al. 2014). Reactions were run using iTaq universal

126 probes Supermix (Bio-Rad, Hercules, CA), $400 \mathrm{nM}$ primers, $200 \mathrm{nM}$ probe, and $500 \mathrm{ng}$ of

127 template spleen DNA in a total volume of $25 \mu$. Cycling conditions consisted of an initial $95^{\circ} \mathrm{C}$

128 step for $3 \mathrm{~min}$, followed by 45 cycles of $95^{\circ} \mathrm{C}$ for $5 \mathrm{~s}$ and $62^{\circ} \mathrm{C}$ for $30 \mathrm{~s}$. Reactions were run in a

129 96-well format on a CFX Connect real-time system (Bio-Rad, Hercules, CA). LruGHV1 plasmid

130 standards for quantitation were prepared by cloning the portion of the $g B$ gene amplified by the

131 degenerate pan-GHV PCR into pCR4-TOPO using a TOPO TA cloning kit (Troyer et al. 2014).

132 Dilutions of plasmids ranging from $10^{7}$ to $10^{2}$ copies per reaction mixture were prepared in a 
133 background of salmon sperm DNA equivalent to $250 \mathrm{ng}$ per reaction mixture, and these

134 standards were run in duplicate in all qPCR runs. Amplification efficiency ranged between 95

135 and $103.6 \%$ for all qPCR runs. Spleen DNA samples were tested in duplicate, and an individual

136 sample was considered positive only if both replicates were positive with greater than 3 copies

137 per reaction. For quantitation of the number of copies per million cells, the number of cell

138 equivalents for each DNA sample was determined as described previously (Terwee et al. 2008;

139 Troyer et al. 2014).

140 PCR amplification and sequencing of $g B$ gene

A nested PCR amplification of the gammaherpesvirus glycoprotein $B(g B)$ gene was

142 performed using the iProof High Fidelity DNA polymerase system (BioRad, Hercules, CA) on a

143 T100 Thermal cycler (BioRad, Hercules, CA). In the first round, the PGHV-F5 forward

144 (GGGGATGTGATTTCGGTGAC) and PGHV-R5 reverse (TCGACCACCTCAAAGTCAATG)

145 primers were used to amplify a $368 \mathrm{bp}$ region of the $g B$ gene from spleen DNA samples. Each

146 reaction contained $100 \mathrm{ng}$ of spleen DNA in a $50 \mu \mathrm{l}$ reaction of $1 \mathrm{X}$ iProof HF Buffer, $200 \mu \mathrm{M}$

147 dNTP mix, $0.5 \mu \mathrm{m}$ PGHV-F5 forward primer, $0.5 \mu \mathrm{m}$ PGHV-R5 reverse primer, $1.0 \mathrm{mM} \mathrm{MgCl}_{2}$,

1480.5 units of iProof DNA polymerase, and DNAse/RNAse-free water. In the second round, the

149 PGHV-F6 forward (GCATGAGAGTTCCAGGTCCA) and PGHV-R6 reverse

150 (TGATGAAGGTGTTGAGAGTTGAAA) primers were used to amplify a 258 bp region of the

151 PCR product from round one. Each reaction contained $2 \mu 1$ of round one PCR product in a $50 \mu 1$

152 reaction of $1 \mathrm{X}$ iProof HF Buffer, $200 \mu \mathrm{M}$ dNTP mix, $0.5 \mu \mathrm{m}$ PGHV-F6 forward primer, $0.5 \mu \mathrm{m}$

153 PGHV-R6 reverse primer, $1.0 \mathrm{mM} \mathrm{MgCl}_{2}, 0.5$ units of iProof DNA polymerase, and

154 DNAse/RNAse-free water. The cycling conditions for both rounds were as follows: $98^{\circ} \mathrm{C}$ for 2

$155 \mathrm{~min}$, followed by 35 cycles of $98^{\circ} \mathrm{C}$ for $30 \mathrm{~s}, 68^{\circ} \mathrm{C}$ for $30 \mathrm{~s}$, and $72^{\circ} \mathrm{C}$ for $30 \mathrm{~s}$, with a final 
156 extension step of $72^{\circ} \mathrm{C}$ for $5 \mathrm{~min}$. PCR product $(4 \mu \mathrm{l})$ from round two was run on a $2 \% \mathrm{TAE}$

157 agarose gel stained with Gel Red DNA dye (Biotium, Inc., Fremont, CA) and visualized under

158 ultraviolet light.

For PCR products showing a single, clear band of the expected size after the two rounds of amplification, a single-step enzymatic cleanup was used to eliminate unincorporated primers and dNTPs prior to sequencing (ExoSAP-IT for PCR Product Cleanup, Affymetrix, Santa Clara, CA). Samples were shipped to Macrogen Corporation (Rockville, MD) for single primer extension, dye-terminator, capillary sequencing on an ABI 3730XL DNA Analyzer (Applied Biosystems, Foster City, CA). Each sample was sequenced in both directions and sequencing results were aligned using MEGA7 version 7.0.14 (Kumar et al. 2016). Consensus $g B$ sequences for each Vermont bobcat were compared to $g B$ sequences obtained previously from Florida,

167 Colorado, and California bobcats (Troyer et al. 2014).

168
Data and statistical analysis

Overall, sex-specific, and age-specific GHV prevalence estimates were calculated by dividing the total number of bobcats with spleen samples containing GHV DNA, as assessed by the $g B$ qPCR assay, by the total number of bobcats tested. The $90 \%$ confidence intervals (CI) for these prevalence estimates were calculated using JMP 10.0.0. The statistical significance of sex differences in GHV prevalence was assessed using counts of positive and negative animals of each sex in $2 \times 2$ contingency tables assessed by a Pearson chi-square test, as implemented in JMP 10.0.0. Statistical significance of differences in GHV prevalence between age categories and between seasons of collection were assessed using the Pearson chi-square test in JMP 10.0.0. Differences in the median viral load between the sexes and age groups were assessed with the nonparametric Wilcoxon rank sum test in JMP 10.0.0. 


\section{Results}

Spleen DNA samples from 64 Vermont bobcats were tested for the presence of GHV

DNA using a qPCR assay targeting the viral $g B$ gene. Overall, $64.1 \%(90 \% \mathrm{CI}=53.8-73.2 \%)$ of

the 64 bobcats were positive for GHV via PCR, and infected animals were identified throughout

the state of Vermont (Figure 1). After stratifying by sex, males showed a $57.1 \%(90 \% \mathrm{CI}=41.8$ -

$71.2 \%)$ prevalence while $69.4 \%$ of females were PCR positive $(90 \% \mathrm{CI}=55.8-80.3 \%)($ Table 1$)$.

This difference was not statistically significant $(\chi 2=1.035, \mathrm{df}=1, \mathrm{P}=0.31)$. GHV detection was

186

187 nearly two-fold higher in 24 adult bobcats $(91.7 \%, \mathrm{CI}=77.7-97.2 \%)$ compared to the 39 young bobcats $(46.2 \%, \mathrm{CI}=33.7-59.1 \%)$ (Table 1$)$, and this difference in prevalence between age categories was statistically significant $(\chi 2=13.277, \mathrm{df}=1, \mathrm{P}=0.00027)$. GHV prevalence in spleen samples collected in the 2015 season was similar to that observed in samples collected in the 2016 season $(\chi 2=0.405, \mathrm{df}=1, \mathrm{P}=0.52)($ Table 1$)$.

In the $41 \mathrm{GHV}$-positive samples, the median viral load, calculated as the number of GHV DNA genomes per million host cells, was 1,590 copies $/ 10^{6}$, with a range of 52 to 27,931 copies $/ 10^{6}$. Male bobcats $(\mathrm{n}=16)$ displayed a higher median viral load $\left(2,296\right.$ copies $\left./ 10^{6}\right)$ and a narrower range (293-21,081 copies $\left./ 10^{6}\right)$ compared to females $(n=25$; median: 1329 ; range: 5227,931 copies $/ 10^{6}$ ) (Figure 2), but the difference between males and females was not statistically significant (Wilcoxon rank sum test, $\mathrm{Z}=1.35, \mathrm{P}=0.18$ ). Median GHV viral load was nearly 2-fold greater in young bobcats $\left(n=18 ; 2,866\right.$ copies $\left./ 10^{6}\right)$ compared to adult bobcats $(n=22 ; 1,499$ copies $/ 10^{6}$ ) (Figure 2). This difference was not significant at the $\mathrm{P}=0.05$ level, but demonstrated an interesting trend towards higher loads in younger animals (Wilcoxon rank sum test, $Z=1.67$, $\mathrm{P}=0.095)$. 
202 GHV $g B$ gene open reading frame was sequencing in five GHV-positive individuals. The $g B$

203 sequence obtained was identical in all five Vermont bobcats and was 99.6\% similar (257/258

204 bases) at the nucleotide level to the published LruGHV1 sequence (KF840715) from a California

205 bobcat, with the single nucleotide difference corresponding to an $\mathrm{I}>\mathrm{C}$ amino acid substitution at

206 codon 108 (Figure 3). The $g B$ sequence from the Vermont bobcats was an exact match to that

207 described previously in Florida bobcats but differed from CA and CO isolates by 1 or 2

208 nucleotides (Figure 3) (Troyer et al. 2014). 


\section{Discussion}

In this study, we detected the presence of gammaherpesvirus DNA in $64 \%$ of the Vermont bobcat spleen DNA samples studied using a sensitive qPCR assay. Sex was not a significant predictor of GHV infection status in our study nor in a previous study of bobcats (Troyer et al. 2014), which is consistent with recent findings that sex of wild and domestic cats was inconsistently predictive of exposure to directly transmitted pathogens but not vector or environmental pathogens (Carver et al. 2016). This finding is in stark contrast to findings in domestic cats, which with the exception of cats in Singapore, have shown a significantly higher prevalence in male versus female cats (Beatty et al. 2014; Ertl et al. 2015; McLuckie et al. 2016).

A significant positive association between age and GHV prevalence was observed in Vermont bobcats, which is consistent with previous observations in bobcat populations in California, Colorado, and Florida (Troyer et al. 2014). In addition to GHV, this pattern of higher rates of infection in older animals has been observed for directly transmitted (e.g. feline immunodeficiency virus), vector-borne (e.g. Bartonella sp.), and environmentally transmitted (e.g. Toxoplasma gondii) pathogens in wild felids (Bevins et al. 2012; Carver et al. 2016). This finding may reflect the cumulative number of potential exposure events experienced by older bobcats during their lifetimes, and is highly suggestive that LruGHV1 is transmitted horizontally during adulthood versus vertically or during the perinatal period.

In contrast to the higher risk of infection in older bobcats, we observed a trend towards a higher viral load in young bobcats, which may reflect the timing of the initial infection event, the transition to latency by GHV, or developmental differences in immunocompetency. In human EBV infection, viral load in peripheral B cells peaks during primary infection, rapid declines after host immune response, and continues a slow decline until reaching the low levels typical of 
232 latency (Fafi-Kremer et al. 2005; Hochberg et al. 2004). A similar pattern was observed in viral

233 load in the spleens of mice infected with murine GHV68 (Olivadoti et al. 2007). Assuming that

234 younger bobcats had more recently experienced primary infection than older ones, higher viral

235 loads would be expected in the younger versus older individuals based upon these observations

236 in other GHV infections. Our observed trend of higher viral load in younger bobcats should

237 interpreted with caution as bobcats were sampled without regard to age in this study. To confirm

238 the trend, future studies should be designed to explicitly compare viral loads in animals of known

239 age, sex, and cause of death, so as to minimize the effect of confounding variables, such as

240 autolysis or unbalanced sampling.

Identification of LruGHV1 in a northeastern US bobcat population adds to previous

reports of PCR detection of LruGHV1 in blood of western (Colorado and California) and

southeastern (Florida) bobcat populations (Troyer et al. 2014), and PCR detection of LruGHV1

from spleen DNA from Colorado (Lozano et al. 2015). We confirm a widespread viral range

across the continental United States, with the highest PCR prevalence estimates being observed

in eastern US populations, Vermont (64\%) and Florida (76\%). Prevalence based upon PCR

247 analysis of blood was $25 \%$ in Colorado and $37 \%$ in California (Troyer et al. 2014). In contrast,

248 PCR analysis of Colorado bobcat spleen DNA (Lozano et al. 2015) produced a prevalence

249 estimate (62\%) more similar to that detected in Vermont, indicating that analysis of spleen DNA

may provide a more sensitive substrate for PCR detection, an observation supported by research

251 showing that murine GHV68 latency is preferentially maintained in splenic B cells (Flano et al.

252 2002). The prevalence estimate in this study may underestimate actual levels of infection in VT

253 bobcats. First, it is feasible that serologic evaluation of LruGHV1 would document an even

254 greater prevalence of this infection in wild populations, given that FcaGHV1 PCR analysis of 
255 domestic cats only detected viral genomes in half of the cats that had seroreactivity against

256 FcaGHV1 antigens (Stutzman-Rodriguez et al. 2016). Second, although severely autolyzed

257 carcasses were not included in this study, some DNA degradation may have occurred due to

258 autolysis prior to sample collection and storage at $-80 \mathrm{C}$ resulting in a lower probability of qPCR

259 detection of LruGHV1.

260 The partial $g B$ gene sequence obtained from Vermont bobcats was identical to that

261 observed from Florida bobcats and closely related to western strains that have been examined.

262 This preliminary data shows more similarity among LruGHV1 isolates across eastern

263 populations, and suggests that this pathogen may be useful to resolve geographic movements and

264 population refugia, similar to the use of feline immunodeficiency virus sequence as a marker for

265 these variables (Antunes et al. 2008; Fountain-Jones et al. 2017; Lee et al. 2012). However, this

266 preliminary interpretation is based solely on patterns observed in a 258 basepair region of the

267 LruGHV1 $g B$ gene and needs to be confirmed by sequencing more of the GHV genome in a

268 larger number of bobcats from the different US populations.

269 The current GHV strain distribution reflects the expansion of bobcat populations from

270 separate Pleistocene refugia in the southeast and northwest regions of the United States. Eastern

271 and western US bobcat populations show strong genetic differentiation in mitochondrial and

272 microsatellite markers which is thought to be the result of expansion from these refugia (Croteau

273 et al. 2012; Reding et al. 2012). Thus, the GHV strains infecting current bobcat populations may

274 be the result of adaptation to the unique genetic structure of the eastern and western populations,

275 or may reflect the historical migration and range expansion events. Since GHV is apparently

276 present in a much wider range of individuals than FIV (which has only been reported in Florida 
277 and California bobcats), it may provide an excellent marker to further explore the relationship of 278 populations across bobcat natural ranges.

279 


\section{Conclusions}

281 We document for the first time the presence of gammaherpesvirus in a population of wild

282 felids living in the northeast United States. The high prevalence of the LruGHV1 strain in

283 Vermont bobcats, combined with previous reports of the strain in western and southeast

284 populations, suggests that the virus has a broad geographic distribution across the continental

285 US. Our results show that older bobcats are more likely to be infected, but may potentially

286 harbor lower viral loads, than young bobcats. We confirm that viral infection status is not

287 associated with sex of bobcats, in contrast to the male-biased pattern of infection observed in

288 domestic cats. These results suggest that horizontal transmission during adulthood is the most

289 likely mechanism of gammaherpesvirus spread in wild bobcats. The sequence differences

290 observed in the GHV $g B$ gene in different bobcat populations may reflect historical migration

291 and range expansion events, but this hypothesis should be tested by analyzing more of the GHV

292 genome in a larger number of infected individuals. 


\section{Acknowledgments}

294 We thank the Vermont Department of Fish and Wildlife, Vermont hunters and trappers, and the 295 Saint Michael's College Biology Department, Office of the Dean of the College, and Office of 296 the Vice President of Academic Affairs for support of the project. We also thank the Saint

297 Michael's College fall 2017 BI-365 class for helpful discussions. The contents of this publication 298 are solely the responsibility of the authors and do not necessarily represent the official views of 299 the NIGMS or NIH. 
301

302

303

304

305

306

307

308

309

310

311

312

313

314

315

316

317

318

319

320

321

322

323

324

325

326

327

328

329

330

331

332

333

334

335

336

337

338

339

340

341

342

343

344

345

346

347
Literature Cited

Ackermann M. 2006. Pathogenesis of gammaherpesvirus infections. Vet Microbiol 113:211-222. 10.1016/j.vetmic.2005.11.008

Antunes A, Troyer JL, Roelke ME, Pecon-Slattery J, Packer C, Winterbach C, Winterbach H, Hemson G, Frank L, Stander P, Siefert L, Driciru M, Funston PJ, Alexander KA, Prager KC, Mills G, Wildt D, Bush M, O'Brien SJ, and Johnson WE. 2008. The evolutionary dynamics of the lion Panthera leo revealed by host and viral population genomics. PLoS Genet 4:e1000251. 10.1371/journal.pgen.1000251

Beatty JA, Troyer RM, Carver S, Barrs VR, Espinasse F, Conradi O, Stutzman-Rodriguez K, Chan CC, Tasker S, Lappin MR, and VandeWoude S. 2014. Felis catus gammaherpesvirus 1; a widely endemic potential pathogen of domestic cats. Virology 460-461:100-107. 10.1016/j.virol.2014.05.007

Bevins SN, Carver S, Boydston EE, Lyren LM, Alldredge M, Logan KA, Riley SP, Fisher RN, Vickers TW, Boyce W, Salman M, Lappin MR, Crooks KR, and VandeWoude S. 2012. Three pathogens in sympatric populations of pumas, bobcats, and domestic cats: implications for infectious disease transmission. PLOS ONE 7:e31403. 10.1371/journal.pone.0031403

Boertje RD, Ellis MM, and Kellie KA. 2015. Accuracy of Moose Age Determinations from Canine and Incisor Cementum Annuli. Wildlife Society Bulletin 39:383-389.

Carver S, Bevins SN, Lappin MR, Boydston EE, Lyren LM, Alldredge M, Logan KA, Sweanor LL, Riley SP, Serieys LE, Fisher RN, Vickers TW, Boyce W, McBride R, Cunningham MC, Jennings M, Lewis J, Lunn T, Crooks KR, and Vandewoude S. 2016. Pathogen exposure varies widely among sympatric populations of wild and domestic felids across the United States. Ecol Appl 26:367-381.

Cesarman E. 2011. Gammaherpesvirus and lymphoproliferative disorders in immunocompromised patients. Cancer Lett 305:163-174. 10.1016/j.canlet.2011.03.003

Cesarman E. 2014. Gammaherpesviruses and lymphoproliferative disorders. Annu Rev Pathol 9:349-372. 10.1146/annurev-pathol-012513-104656

Coleman CB, Nealy MS, and Tibbetts SA. 2010. Immature and transitional B cells are latency reservoirs for a gammaherpesvirus. J Virol 84:13045-13052. 10.1128/JVI.01455-10

Croteau EK, Heist EJ, Nielsen CK, Hutchinson JR, and Hellgren EC. 2012. Microsatellites and mitochondrial DNA reveal regional population structure in bobcats (Lynx rufus) of North America. Conservation Genetics 13:1637-1651. 10.1007/s10592-012-0416-0

Ertl R, Korb M, Langbein-Detsch I, and Klein D. 2015. Prevalence and risk factors of gammaherpesvirus infection in domestic cats in Central Europe. Virol J 12:146. 10.1186/s12985-015-0381-6

Fafi-Kremer S, Morand P, Brion JP, Pavese P, Baccard M, Germi R, Genoulaz O, Nicod S, Jolivet M, Ruigrok RW, Stahl JP, and Seigneurin JM. 2005. Long-term shedding of infectious epstein-barr virus after infectious mononucleosis. J Infect Dis 191:985-989. 10.1086/428097

Flano E, Kim IJ, Woodland DL, and Blackman MA. 2002. Gamma-herpesvirus latency is preferentially maintained in splenic germinal center and memory B cells. J Exp Med 196:1363-1372.

Fountain-Jones NM, Craft ME, Funk WC, Kozakiewicz C, Trumbo DR, Boydston EE, Lyren LM, Crooks K, Lee JS, VandeWoude S, and Carver S. 2017. Urban landscapes can change virus gene flow and evolution in a fragmentation-sensitive carnivore. Mol Ecol 26:6487-6498. 10.1111/mec.14375

Hochberg D, Souza T, Catalina M, Sullivan JL, Luzuriaga K, and Thorley-Lawson DA. 2004. Acute infection with Epstein-Barr virus targets and overwhelms the peripheral memory B-cell compartment with resting, latently infected cells. J Virol 78:5194-5204.

Kaye S, Wang W, Miller C, McLuckie A, Beatty JA, Grant CK, VandeWoude S, and Bielefeldt-Ohmann H. 2016. Role of Feline Immunodeficiency Virus in Lymphomagenesis--Going Alone or Colluding? ILAR J 57:24-33. 10.1093/ilar/ilv047 
Kumar S, Stecher G, and Tamura K. 2016. MEGA7: Molecular Evolutionary Genetics Analysis Version 7.0 for Bigger Datasets. Mol Biol Evol 33:1870-1874. 10.1093/molbev/msw054

Lee JS, Ruell EW, Boydston EE, Lyren LM, Alonso RS, Troyer JL, Crooks KR, and Vandewoude S. 2012. Gene flow and pathogen transmission among bobcats (Lynx rufus) in a fragmented urban landscape. Mol Ecol 21:1617-1631. 10.1111/j.1365-294X.2012.05493.x

Lozano CC, Sweanor LL, Wilson-Henjum G, Kays RW, Moreno R, VandeWoude S, and Troyer RM. 2015. Identification of Novel Gammaherpesviruses in Ocelots (Leopardus pardalis) and Bobcats (Lynx rufus) in Panama and Colorado, USA. J WildI Dis 51:911-915. 10.7589/2015-01-027

McLuckie A, Tasker S, Dhand NK, Spencer S, and Beatty JA. 2016. High prevalence of Felis catus gammaherpesvirus 1 infection in haemoplasma-infected cats supports co-transmission. Vet J 214:117-121. 10.1016/j.tvjl.2016.06.001

O'Toole D, and Li H. 2014. The pathology of malignant catarrhal fever, with an emphasis on ovine herpesvirus 2. Vet Pathol 51:437-452. 10.1177/0300985813520435

Olivadoti M, Toth LA, Weinberg J, and Opp MR. 2007. Murine gammaherpesvirus 68: a model for the study of Epstein-Barr virus infections and related diseases. Comp Med 57:44-50.

Reding DM, Bronikowski AM, Johnson WE, and Clark WR. 2012. Pleistocene and ecological effects on continental-scale genetic differentiation in the bobcat (Lynx rufus). Mol Ecol 21:3078-3093. 10.1111/j.1365-294X.2012.05595.x

Russell GC, Stewart JP, and Haig DM. 2009. Malignant catarrhal fever: a review. Vet J 179:324-335. 10.1016/j.tvjl.2007.11.007

Speck SH, and Ganem D. 2010. Viral latency and its regulation: lessons from the gamma-herpesviruses. Cell Host Microbe 8:100-115. 10.1016/j.chom.2010.06.014

Stutzman-Rodriguez K, Rovnak J, VandeWoude S, and Troyer RM. 2016. Domestic cats seropositive for Felis catus gammaherpesvirus 1 are often qPCR negative. Virology 498:23-30. 10.1016/j.virol.2016.07.027

Tateno M, Takahashi M, Miyake E, Nishigaki K, Tsujimoto H, and Endo Y. 2017. Molecular epidemiological study of gammaherpesvirus in domestic cats in Japan. J Vet Med Sci 79:17351740. 10.1292/jvms.17-0039

Terwee JA, Carlson JK, Sprague WS, Sondgeroth KS, Shropshire SB, Troyer JL, and VandeWoude S. 2008. Prevention of immunodeficiency virus induced CD4+ T-cell depletion by prior infection with a non-pathogenic virus. Virology 377:63-70. 10.1016/j.virol.2008.03.037

Troyer RM, Beatty JA, Stutzman-Rodriguez KR, Carver S, Lozano CC, Lee JS, Lappin MR, Riley SP, Serieys LE, Logan KA, Sweanor LL, Boyce WM, Vickers TW, McBride R, Crooks KR, Lewis JS, Cunningham MW, Rovnak J, Quackenbush SL, and VandeWoude S. 2014. Novel gammaherpesviruses in North American domestic cats, bobcats, and pumas: identification, prevalence, and risk factors. $J$ Virol 88:3914-3924. 10.1128/JVI.03405-13 


\section{Table $\mathbf{1}$ (on next page)}

Sample numbers and prevalence estimates from gammaherpesvirus qPCR analysis of DNA extracted from Vermont bobcat spleens 


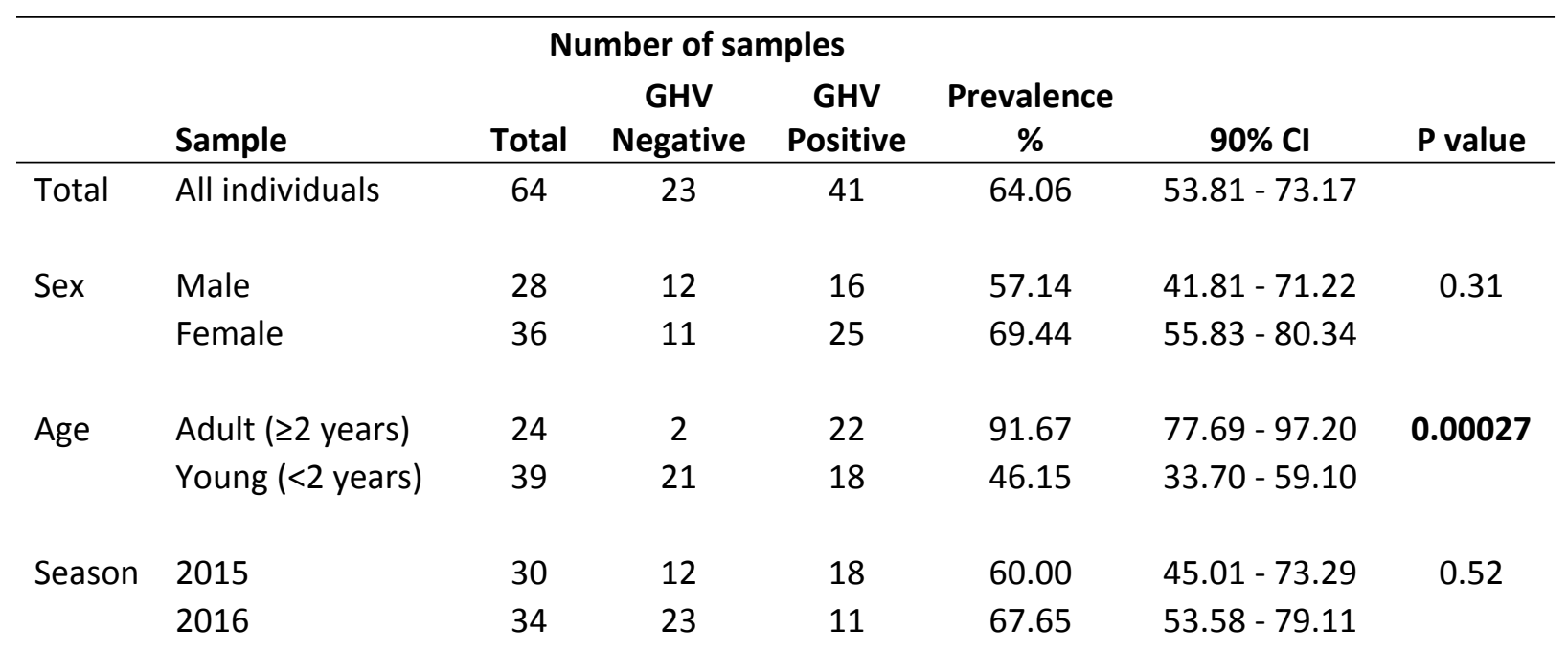

1 


\section{Figure 1}

Geographic distribution of gammaherpesvirus prevalence in Vermont bobcats.

LruGHV1 qPCR-positive bobcats are indicated by green pins and qPCR-negative bobcats are indicated by white pins. Map data (c) 2018 Google. 


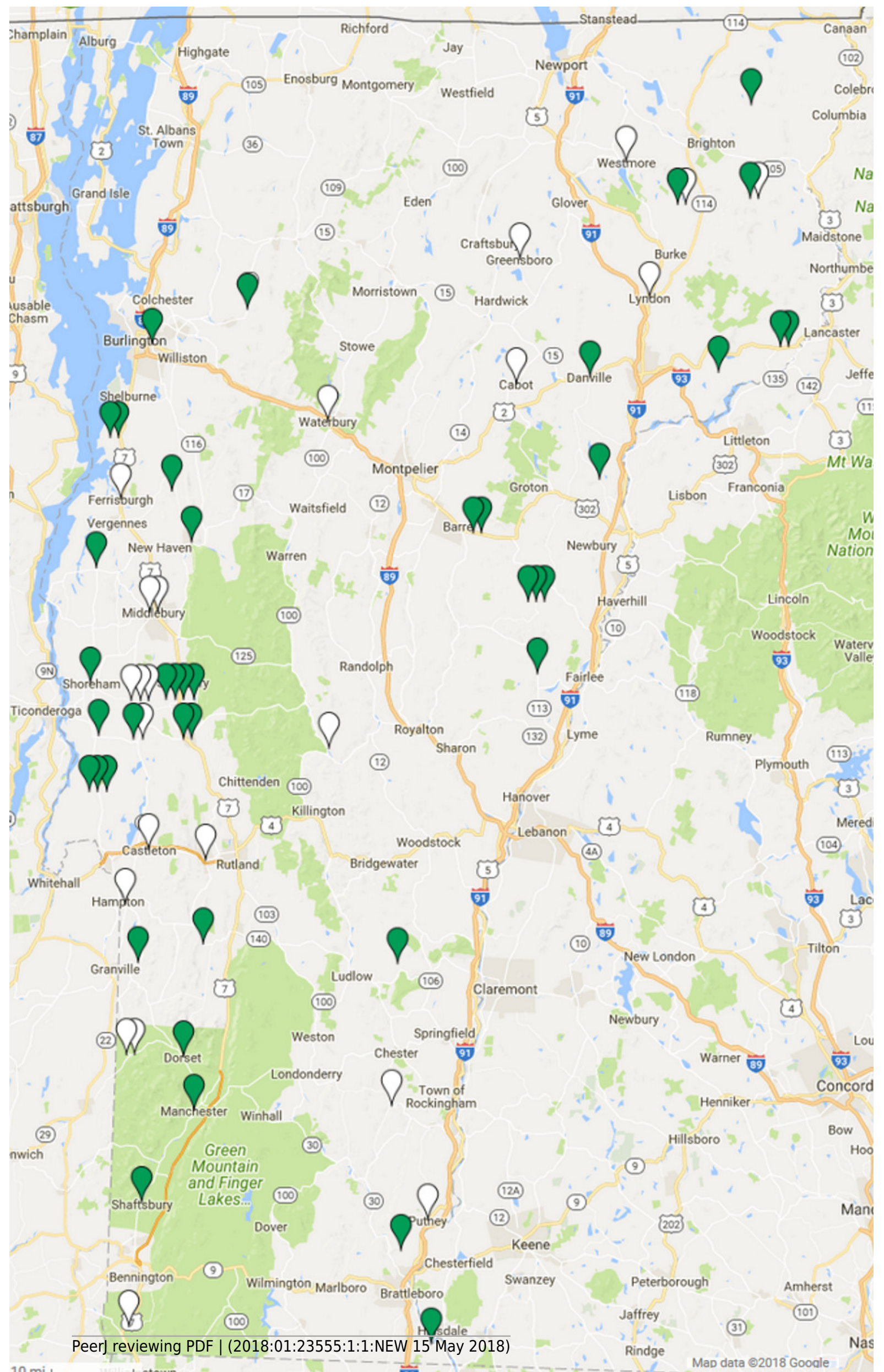


Figure 2

DNA viral load of 41 LruGHV1-positive Vermont bobcats stratified by sex and age.

Age was not available for one GHV-positive female bobcat. Boxes show median values and 25th and 75th percentiles, while whiskers extend an additional $1.5 x$ of interquartile range. Outliers are shown as individual points.

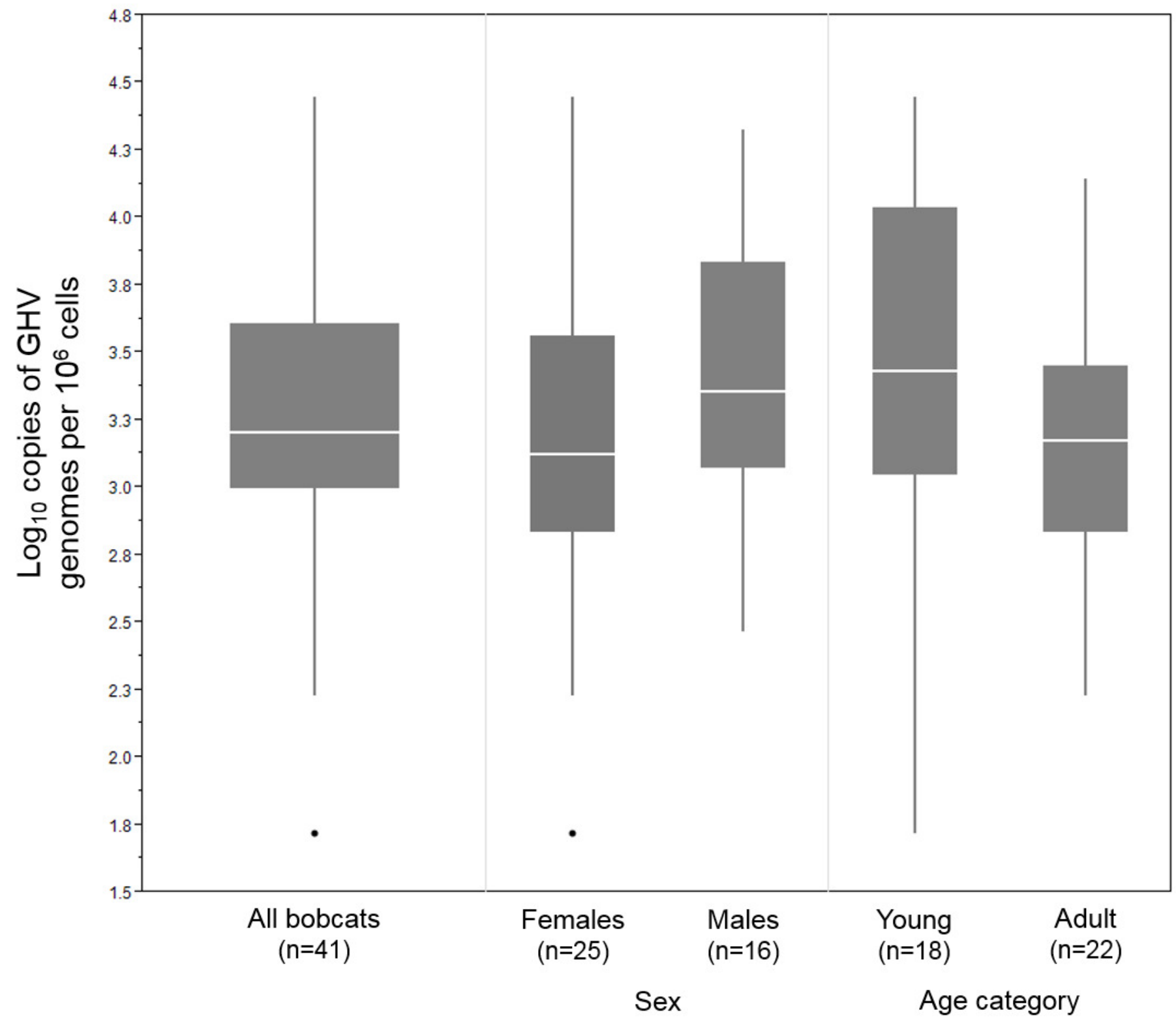


Figure 3 (on next page)

Alignment of partial LruGHV1 glycoprotein $B(g B)$ sequences from four US bobcat populations.

For each isolate, collection location is indicated by two letter state code and animal origin is listed in parentheses. Amino acid-changing substitutions are highlighted in gray with the resultant amino acid in brackets below the codon. The asterisk symbol (*) indicates a stop codon. Amino acid position was based on published LruGHV1 $g B$ sequence from a CA bobcat (GenBank accession number KF840716). 
Amino acid position Amino acid sequence Nucleotide sequence $\mathrm{CA} / \mathrm{CO}$ isolate (x208/×356) $\mathrm{CA}$ isolate $(\mathrm{x} 159)$ $C A$ isolate (x148) CA/CO isolate (x209/x356) FL isolate (x1212)

Amino acid position Amino acid sequence Nucleotide sequence $\mathrm{CA} / \mathrm{CO}$ isolate $(\mathrm{x} 208 / \mathrm{x} 356)$ CA isolate $(x 159)$ $\mathrm{CA}$ isolate (x148) $\mathrm{CA} / \mathrm{CO}$ isolate (x209/x356) FL isolate (x1212) VT isolate (SP043)

Amino acid position Amino acid sequence Nucleotide sequence CA/CO isolate (x208/x356) $\mathrm{CA}$ isolate (x159) CA isolate (x148)

$\mathrm{CA} / \mathrm{CO}$ isolate $(\mathrm{x} 209 / \mathrm{x} 356)$ FL isolate (x1212) VT isolate (SP043)

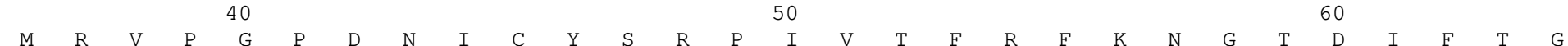
ATG AGA GTT CCA GGT CCA GAC AAC ATA TGC TAC TCC AGA CCC ATC GTC ACC TTC AGG TTT AAA AAC GGG ACT GAC ATC TTC ACT GGA

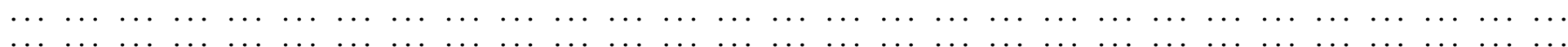
$\begin{array}{lllllllllllllllllllllllll}\ldots & \ldots & \ldots & \ldots & \ldots & \ldots & \ldots & \ldots & \ldots & \ldots & \ldots & \ldots & \ldots & \ldots & \ldots & \ldots & \ldots & \ldots & \ldots & \ldots & \ldots & \ldots & \ldots & \ldots & \ldots\end{array} \ldots$

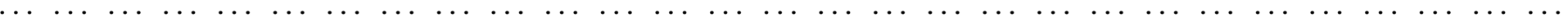

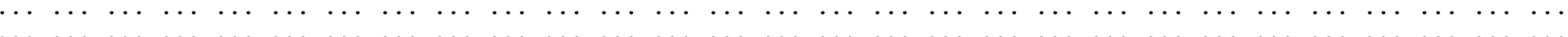

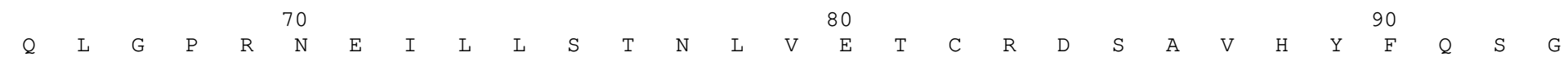
CAA TTG GGT CCT AGA AAT GAA ATT CTA TTA TCA ACA AAC TTA GTG GAG ACT TGT AGA GAC TCT GCT GTT CAC TAC TTT CAG TCA GGG

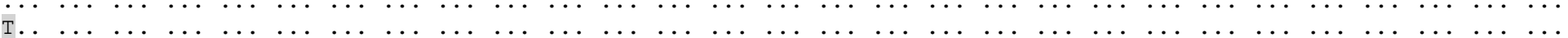

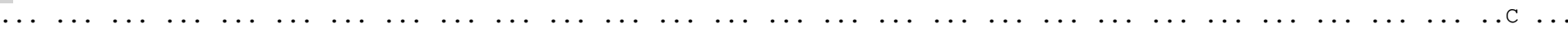

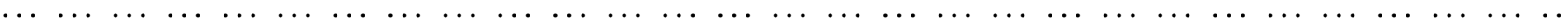
$\begin{array}{llllllllllllllllllllllllllllllllllll}\ldots & \ldots & \ldots & \ldots & \ldots & \ldots & \ldots & \ldots & \ldots & \ldots & \ldots & \ldots & \ldots & \ldots & \ldots & \ldots & \ldots & \ldots & \ldots & \ldots & \ldots & \ldots & \ldots & \ldots & \ldots & \ldots & \ldots & \ldots & \ldots\end{array}$ $[*]$

$\begin{array}{llllllllllllllllllllllllllllllll} & \mathrm{H} & \mathrm{Q} & \mathrm{M} & \mathrm{H} & \mathrm{K} & \mathrm{Y} & \mathrm{V} & \mathrm{N} & \mathrm{Y} & \mathrm{Q} & \mathrm{H} & \mathrm{K} & \mathrm{S} & \mathrm{T} & \mathrm{I} & \mathrm{D} & \mathrm{I} & \mathrm{Q} & \mathrm{N} & \mathrm{F} & \mathrm{S} & \mathrm{T} & \mathrm{L} & \mathrm{N} & \mathrm{T} & \mathrm{F} & \mathrm{I}\end{array}$ CAT CAA ATG CAT AAG TAT GTA AAC TAT CAA CAT AAA AGC ACA ATA GAT ATT CAG AAT TTT TCA ACT CTC AAC ACC TTC ATC

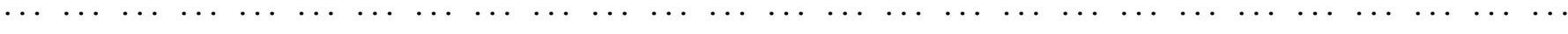
$\begin{array}{llllllllllllllllllllllllllllll}\ldots & \ldots & \ldots & \ldots & \ldots & \ldots & \ldots & \ldots & \ldots & \ldots & \ldots & \ldots & \ldots & \ldots & \ldots & \ldots & \ldots & \ldots & \ldots & \ldots & \ldots & \ldots & \ldots & \ldots & \ldots & \ldots & \ldots \\ \ldots & \ldots & \ldots & \ldots & \ldots & \ldots & \ldots & \ldots & \ldots & \ldots & \ldots & \ldots & \ldots & \ldots & . & \ldots & \ldots & \ldots & \ldots & \ldots & \ldots & \ldots & \ldots & \ldots & \ldots & \ldots & \ldots & \ldots\end{array}$

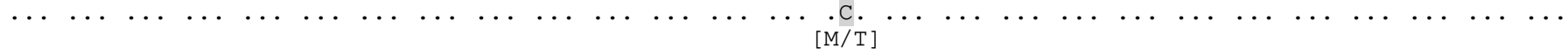

